\title{
Hybrid Plasmonic Biosensor for Simultaneous Measurement of Both Thickness and Refractive Index
}

\author{
Youqiao Ma \\ Technological University Dublin \\ Gerald Farrell \\ Technological University Dublin, gerald.farrell@tudublin.ie \\ Yuliya Semenova \\ Technological University Dublin, yuliya.semenova@tudublin.ie
}

See next page for additional authors

Follow this and additional works at: https://arrow.tudublin.ie/engscheceart

Part of the Bioinformatics Commons

\section{Recommended Citation}

Ma, Y., Farrell, G., Semenova, Y., Chan, H.P. and Wu, Q. Hybrid Plasmonic Biosensor for Simultaneous Measurement of both Thickness and Refractive Index. Infrared Physics \& Technology, vol. 60, pp. 134-136, 2013. http://dx.doi.org/10.1016/j.infrared.2013.04.001.

This Article is brought to you for free and open access by the School of Electrical and Electronic Engineering at ARROW@TU Dublin. It has been accepted for inclusion in Articles by an authorized administrator of ARROW@TU Dublin. For more information, please contact arrow.admin@tudublin.ie, aisling.coyne@tudublin.ie, gerard.connolly@tudublin.ie.

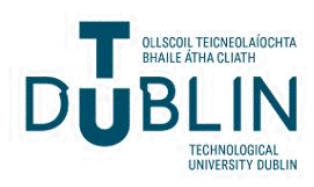




\section{Authors}

Youqiao Ma, Gerald Farrell, Yuliya Semenova, Hau Ping Chan, and Qiang wu 
Provided for non-commercial research and education use. Not for reproduction, distribution or commercial use.

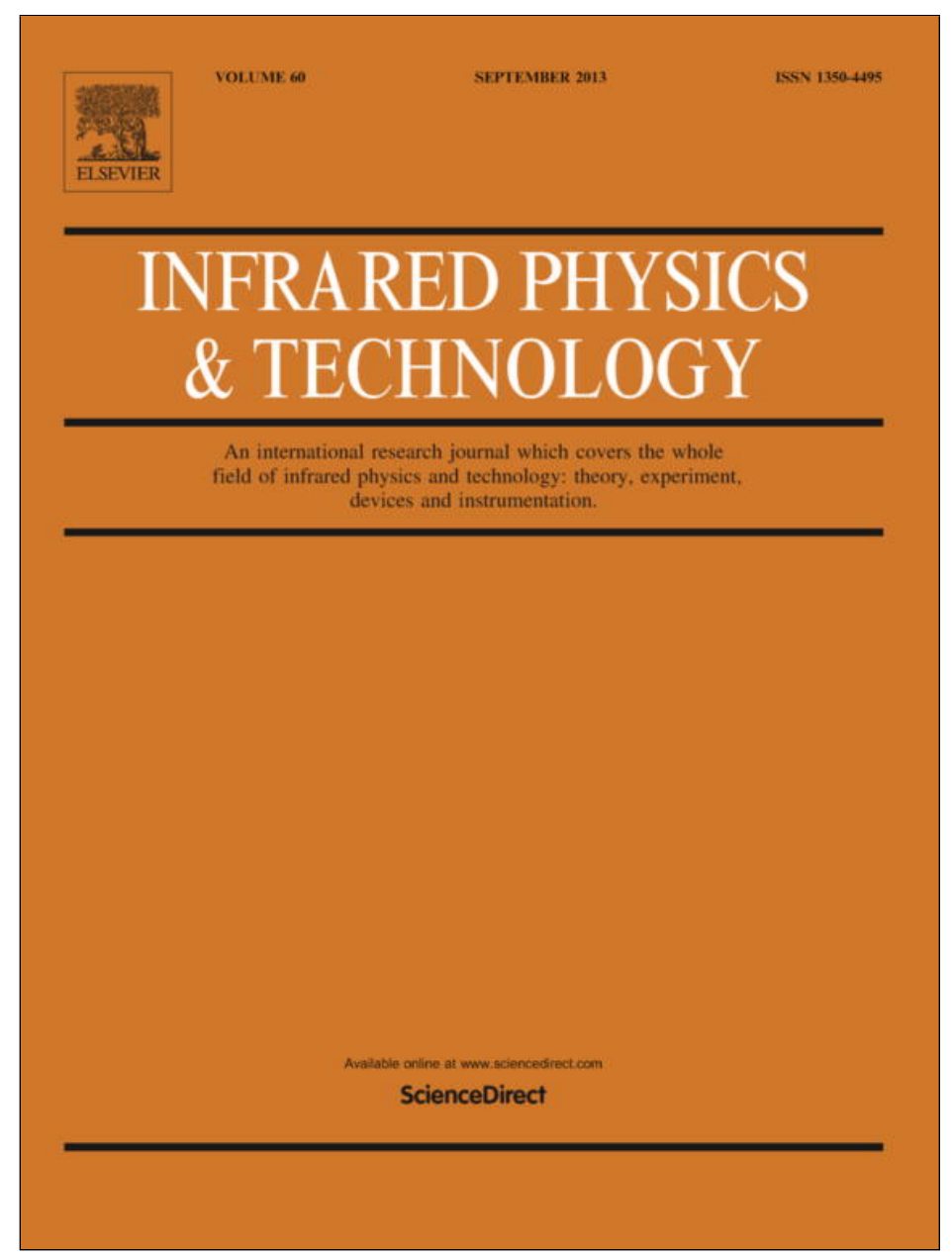

This article appeared in a journal published by Elsevier. The attached copy is furnished to the author for internal non-commercial research and education use, including for instruction at the authors institution and sharing with colleagues.

Other uses, including reproduction and distribution, or selling or licensing copies, or posting to personal, institutional or third party websites are prohibited.

In most cases authors are permitted to post their version of the article (e.g. in Word or Tex form) to their personal website or institutional repository. Authors requiring further information regarding Elsevier's archiving and manuscript policies are encouraged to visit:

http://www.elsevier.com/authorsrights 


\title{
Hybrid plasmonic biosensor for simultaneous measurement of both thickness and refractive index
}

\author{
Youqiao Ma ${ }^{a}$, Gerald Farrell ${ }^{\mathrm{a}}$, Yuliya Semenova ${ }^{\mathrm{a}}$, Hau Ping Chan ${ }^{\mathrm{b}}$, Qiang $\mathrm{Wu}^{\mathrm{a}, *}$ \\ ${ }^{a}$ Photonics Research Center, School of Electronic and Communications Engineering, Dublin Institute of Technology, Kevin Street, Dublin 8, Ireland \\ ${ }^{\mathrm{b}}$ Department of Electronic Engineering, City University of Hong Kong, Hong Kong, China
}

\section{H I G H L I G H T S}

- The proposed structure supports both cavity resonant mode (CRM) and hybrid plasmonic-cavity mode (HPCM).

- CRM is only sensitive to the environmental refractive index (RI).

- HPCM are sensitive to both RI and thickness of analyte layer.

- The proposed structure can measure both RI and thickness of analyte simultaneously.

\section{A R T I C L E I N F O}

\section{Article history:}

Received 12 October 2012

Available online 23 April 2013

\section{Keywords:}

Surface plasmon polaritons (SPPs)

Refractive index sensing

Hybrid plasmonics biosensor

\begin{abstract}
A B S T R A C T
A novel hybrid plasmonic biosensor using a sub-wavelength reflection metal grating is proposed and analyzed. The simulation results show that the hybrid plasmonic-cavity (HPCM) are wavelength dependent on both the refractive index (RI) and thickness of the surrounding analyte layer, with a sensitivity of $448 \mathrm{~nm} / \mathrm{RIU}$ (refractive index unit) and $75 \mathrm{~nm} / \mu \mathrm{m}$, respectively. However wavelength related to the cavity resonant mode (CRM) is only sensitive to the environmental refractive index changes. In principle it is possible to determine both RI and thickness of the coating layer simultaneously.
\end{abstract}

(C) 2013 Elsevier B.V. All rights reserved.
Surface plasmon polaritons (SPPs), confined at a metal-dielectric interface with an exponentially decaying field in both sides, have been considered as energy and information carriers which significantly overcome the conventional diffraction limit at a subwavelength level. A substantial amount of research has been carried out in the visible [1], infrared [2,3], and terahertz regions $[4,5]$. Extraordinary transmission (EOT) phenomena have sparked a lot of interest in metal optics, including EOT through a sub-wavelength single metallic slit [6], slit array [7] and hole array [8]. It is generally believed that the cavity resonant mode (CRM) and the excitation of SPPs play an essential role in EOT phenomena. One type of structure is a reflection metal grating (RMG), for which there have been some theoretical studies, but only in connection with an investigation of SPPs and/or localized SPPs (LSPPs) $[9,10]$. Recently, we have investigated the characteristics of SPPs waves on a RMG with perpendicular slits using a transmission line model [11]. The motivation for studying RMG is that a wide variety of optical devices based on RMG structures have been proposed, including photovoltaic cells [12], plasmonic sensors [13], and so on. In addition it is known that layer-by-layer assemblies based on deoxyribonucleic acid (DNA) hybridization have significant po-

\footnotetext{
* Corresponding author. Tel.: +35314024569.

E-mail address: qiang.wu@dit.ie (Q. Wu).
}

tential for various biochemical nanotechnologies [14]. In such applications, it is very important to be able to achieve real-time detection of the physicochemical properties of the layers i.e. their thickness and refractive index (RI).

In this paper, a novel biosensor consisting of sub-wavelength reflection RMG is proposed and characterized by the Finite Element Method (FEM) [15]. The simulation results show that the bandwidth of the reflection dip excited by the hybrid plasmonic-cavity mode (HPCM) is much narrower than that of CRM and its spectral response is very sensitive to both the refractive index and the thickness of the dielectric layer while the CRM is only sensitive to the refractive index of the dielectric layer. Such characteristics indicate that the proposed structure can be used to solve the problem of cross sensitivity of HPCM and it is particularly suitable for biochemical applications for the detection of both refractive index and the thickness of organic materials.

The schematic diagram of the investigated structure is shown in Fig. 1, which consists of a one-dimensional sub-wavelength RMG (with the grating period $T$, the slit width $f$ and height $h$ ) coated with a dielectric layer (i.e. analyte, with a thickness $d$ and refractive index $n_{d}$ ) surrounded by air. For this structure, it is assumed that a source with TM-polarization (magnetic field vector parallel to the grating slits) normally illuminates the grating. For the purpose of the simulation, we assume the slit is filled with a dielectric 


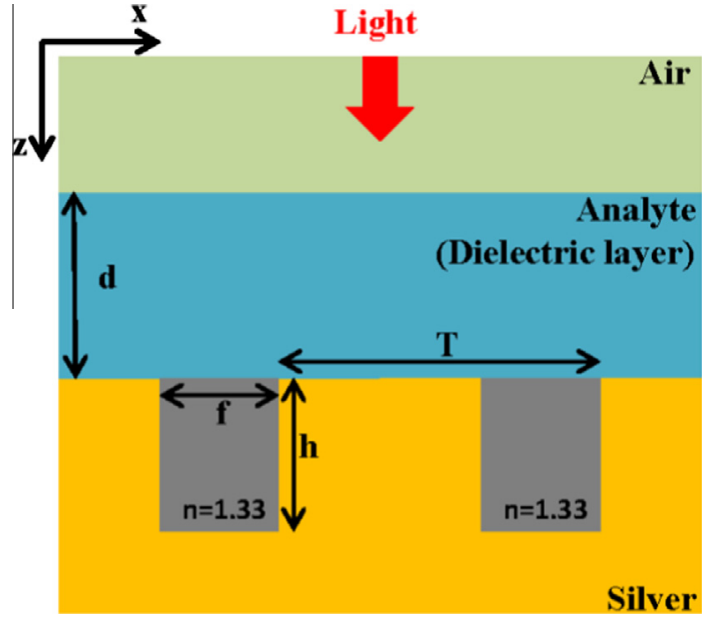

Fig. 1. Schematic diagram of the plasmonic grating used as a biosensor illustrating the structural parameters and coordinates. The detecting layer is denoted as the dielectric layer with a thickness of $d$ and RI of $n_{d}$.

material with a RI ( $n=1.33)$ close to that of the analyte. In the simulation, an extend Debye dispersion model is used to characterize the frequency-dependent dielectric constant of metal $\varepsilon_{m}$ [16]:

$\varepsilon_{m}(\omega)=1-\sum_{n=1}^{6} \frac{\Delta \varepsilon_{n}}{a_{n} \omega^{2}+i b_{n} \omega-c_{n}}$

where $\omega$ is the angular frequency of the incident electromagnetic radiation, and for silver, the related values of $\Delta \varepsilon_{n}, a_{n}, b_{n}$ and $C_{n}$ are employed as follows: $\Delta \varepsilon_{1}=1759.471, \Delta \varepsilon_{2}=135.344, \Delta \varepsilon_{3}=$ 258.1946, $\Delta \varepsilon_{4}=22.9046, \Delta \varepsilon_{5}=1749.06, \Delta \varepsilon_{6}=11756.18, a_{1}=a_{2}=$ $a_{3}=a_{4}=a_{5}=a_{6}=1, \quad b_{1}=0.243097, \quad b_{2}=19.68071, \quad b_{3}=2.289161$, $b_{4}=0.329194, \quad b_{5}=4.639097, \quad b_{6}=12.25, \quad C_{1}=0, \quad C_{2}=17.07876$, $C_{3}=515.022, C_{4}=1718.357, C_{5}=2116.092$ and $C_{6}=10559.42$.

Fig. 2a shows the simulated reflective spectra for the structure as shown in Fig. 1. The structural parameters in the simulation are as follows: $n_{d}=1.33, T=600 \mathrm{~nm}, f=50 \mathrm{~nm}, h=300 \mathrm{~nm}$ and $d=600 \mathrm{~nm}$. As shown in Fig. 2a there are basically two reflection dips in the wavelength range from $700 \mathrm{~nm}$ to $1000 \mathrm{~nm}$, positioned at $773.2 \mathrm{~nm}$ and $930 \mathrm{~nm}$, respectively. The width of the reflection dip at $930 \mathrm{~nm}$ is much wider than that at $773.2 \mathrm{~nm}$. The $Q$ value for the dip at $930 \mathrm{~nm}$ is 33.1 , which is less than twice the $Q$ value of 72.8 for the dip at $773.2 \mathrm{~nm}$.

It is useful to visualize the field distributions within the structure for different wavelengths and resonance types. Fig. $2 \mathrm{~b}$ and $\mathrm{c}$ shows the corresponding normalized magnetic $2 \mathrm{D}$-field patterns. Fig. $2 \mathrm{~b}$ indicates that the reflection dip at $930 \mathrm{~nm}$ is excited by CRM since the magnetic fields are strongly concentrated in the slits with standing wave patterns, and only a small portion of the magnetic field leaks to outside. Fig. 2c shows that the reflection dip at $773.2 \mathrm{~nm}$ is excited by HPCM i.e. the mode is of hybrid nature with contributions of SPPs and CRM. Often one contribution is, however, dominant.

Based on insights provided in Fig. 2, in the presence of periodic slits, the light will be diffracted by the slits. The wave-vector of the diffracted light is superimposed on the incident light. The SPPs can be excited only when the mismatched wave-vector is bridged: $k_{S P P S}=k_{\text {in }} \sin \alpha \pm 2 n \pi / T$, where $k_{S P P S}$ denotes the wave-vector of SPPs, $k_{i n}, \alpha$ and $n$ are the wave-vector of the incident light, the angle of incidence and an integer not less than zero, respectively.

The highly concentrated field at the analyte layer provides a very effective overlap between HPCM and surrounding analyte, leading to the conclusion that HPCM may be an ideal candidate for the biosensing applications.
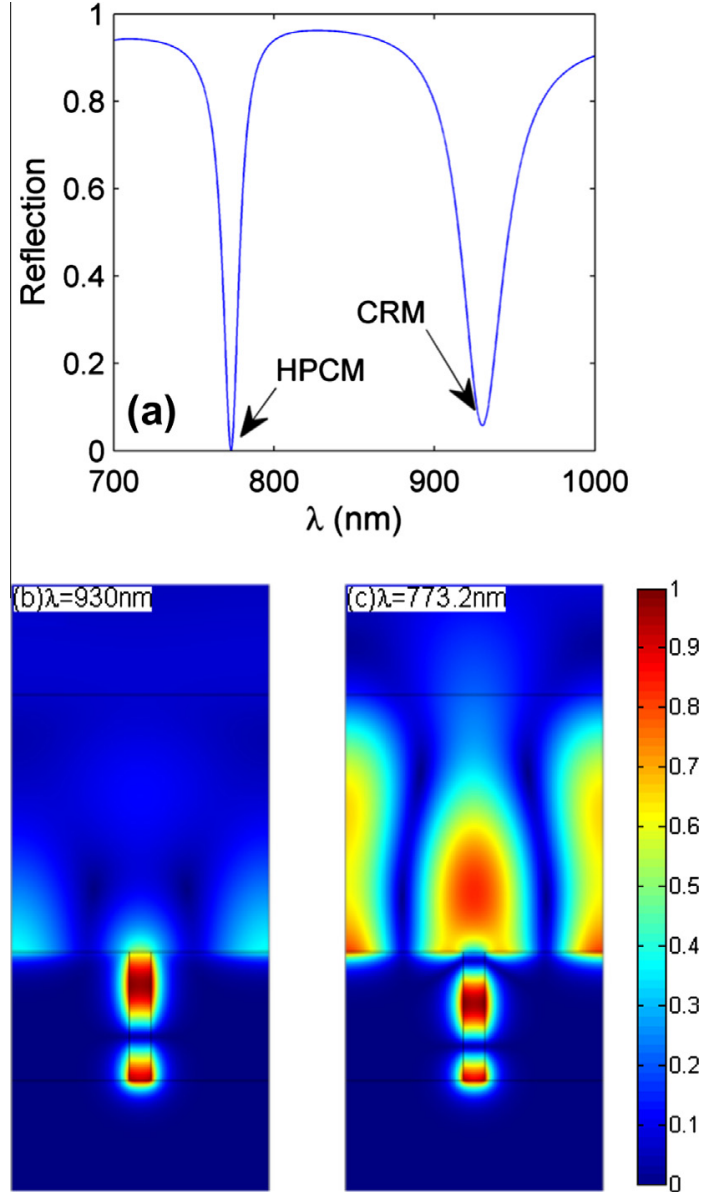

Fig. 2. (a) Reflection spectrum at normal incidence of the investigated structure. Two reflection dips occur at $930 \mathrm{~nm}$ and $773.2 \mathrm{~nm}$ in the investigated wavelength range. The normalized magnetic field distributions of radiation at a wavelength of (b) $930 \mathrm{~nm}$ and (c) $773.2 \mathrm{~nm}$ with normal incidence.

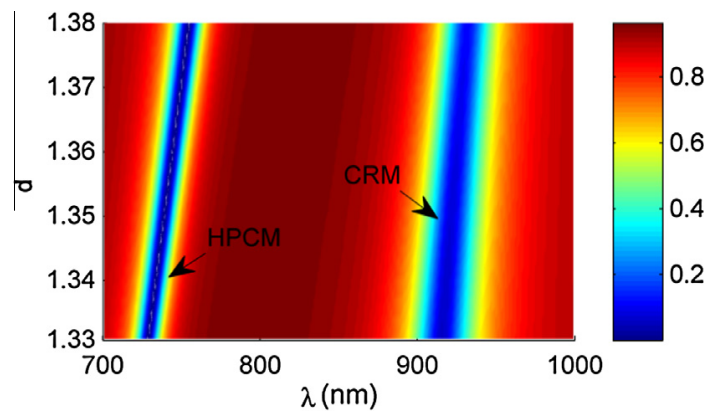

Fig. 3. Contour plot of the normalized reflection versus both the wavelength and the refractive index of $n_{d}$ for the investigated structure. Both the reflection dips associated with HRCM and CRM are sensitive to the variations of $n_{d}$.

Fig. 3 shows a contour plot of the normalized reflected intensity as a function of both wavelength and the value of $n_{d}$ as $n_{d}$ varies from 1.33 to 1.38 . The red region indicates a total reflection and the dark blue streak regions represent reflectance dips arising from the excitation of HPCM or CRM. The geometric parameters are $T=600 \mathrm{~nm}, f=50 \mathrm{~nm}, h=300 \mathrm{~nm}$ and $d=600 \mathrm{~nm}$. It is clear that the shift in the reflective dip related to HPCM is more sensitive to $n_{d}$ than that of another dip produced by the CRM. The reflective dip related to the CRM experiences a linear red shift with a sensitivity $(\Delta \lambda / \Delta n)$ of $240 \mathrm{~nm} / \mathrm{RIU}$ (refractive index unit). However the dip associated with HPCM experiences a red shift with a 


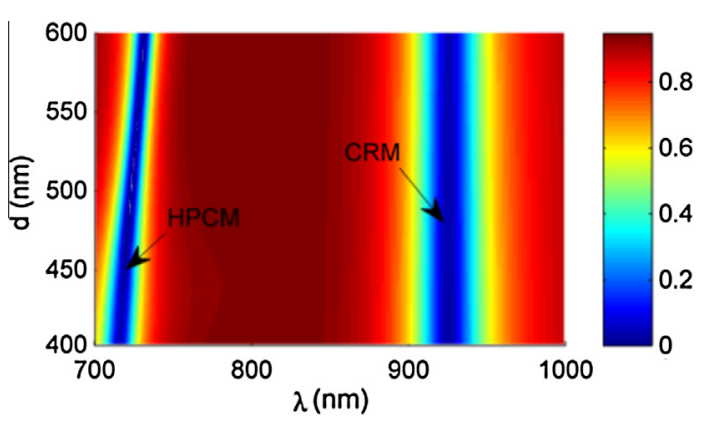

Fig. 4. Contour plot of the normalized reflection versus both the wavelength and the thickness of the analyte layer $d$ for the investigated structure. There is a clear variation of the reflection dip related to HPCM as $d$ changes, while CRM remains unchanged with changes in $d$.

significantly higher sensitivity of $448 \mathrm{~nm} / \mathrm{RIU}$. This is due to the fact that the magnetic field of CRM is more concentrated within the cavity which is relatively far from the analyte layer compared to that of HPCM and hence the influence of analyte layer on HPCM is more significant than that on CRM.

The influence of the thickness of the analyte layer on the spectral response was also investigated and shown in Fig. 4. The parameters used in the simulation are $T=600 \mathrm{~nm}, f=50 \mathrm{~nm}, h=300 \mathrm{~nm}$ and $n_{d}=1.35$. As expected as the thickness $d$ varies, the reflective dips related to the CRM remains unchanged, while the dip related to the HPCM experiences significant linear red-shift with a sensitivity $(\Delta \lambda / \Delta d)$ of $75 \mathrm{~nm} / \mu \mathrm{m}$.

Within the scope of the above discussions, we know that the central wavelength of the dip related to HPCM depends on both the thickness and refractive index seriously, however the wavelength shift related to CRM is thickness independent but sensitive to refractive index, making it possible to use the technique proposed in the paper to distinguish and measure both the thickness and RI simultaneously. As an example in our simulation, the relationship between HPCM-induced wavelength shift $\left(\Delta \lambda_{\text {HPCM }}\right)$ and CRM-induced spectral response variation $\left(\Delta \lambda_{C R M}\right)$ can be expressed as:

$$
\left\{\begin{array}{l}
\Delta \lambda_{\mathrm{HPCM}}=448 \frac{\mathrm{nm}}{R I U} * \Delta n_{d}+75 \frac{\mathrm{nm}}{\mu \mathrm{m}} * \Delta d \\
\Delta \lambda_{\mathrm{CRM}}=240 \frac{\mathrm{nm}}{\mathrm{RIU}} * \Delta n_{d}
\end{array}\right.
$$

where $\Delta d$ and $\Delta n_{d}$ are the thickness and RI variations of the analyte layer, respectively. Since the wavelength variations of two spectral dips can be measured independently, by solving Eq. (2), both the thickness and RI can be determined independently.

In conclusion, we have proposed and theoretically analyzed a novel hybrid plasmonic biosensor based on a sub-wavelength reflection metal grating. Our investigations show that the wavelength dip related to HPCM has a higher $Q$ value of 72.8 compared to the other dip due to a CRM with $Q$ value of 33.1. The sensitivity of the dip related to HPCM to the refractive index of the analyte layer is approximately twice than that of the dip related to CRM. The sensitivity of HPCM to the analyte layer thickness means that HPCM can be used to detect the thickness of a coating layer (i.e. an organic analyte) with a sensitivity of $75 \mathrm{~nm} / \mu \mathrm{m}$. Finally by measuring the spectral response shifts of both HPCM and CRM dips, the proposed technique can be used to detect both the RI and the thickness of the specific biochemical targets or analytes simultaneously.

This work was supported by Dublin Institute of Technology under the Fiosraigh Research Scholarship, Science Foundation Ireland under Grant Nos. 11/TIDA/B2051, 07/SK/I1200, 07/SK/I1200STTF1 1 and the Open Fund of the State Key Laboratory of Information Photonics and Optical Communications (Beijing University of Posts and Telecommunications), PR China.

\section{References}

[1] X.Y. He, Comparison of the waveguide properties of gap surface plasmon in the terahertz region and visible spectra, J. Opt. A: Pure Appl. Opt. 11 (4) (2009) 045708.

[2] N. Liu, M. Mesch, T. Weiss, M. Hentschel, H. Giessen, Infrared perfect absorber and its application as plasmonic sensor, Nano. Lett. 10 (7) (2010) 2342-2348.

[3] Y.Q. Ma, G. Farrell, Y. Semenova, Q. Wu, Analysis and applications of nanocavity structures used as tunable filters and sensors, Infrared Phys. Technol. 55 (5) (2012) 389-394.

[4] X.Y. He, Numerical analysis of the propagation properties of subwavelength semiconductor slit in the terahertz region, Opt. Express 17 (17) (2009) 1535915371.

[5] X.Y. He, Q.J. Wang, S.F. Yu, Numerical study of gain-assisted terahertz hybrid plasmonic waveguide, Plasmonics 7 (3) (2012) 571-577.

[6] Y.X. Cui, S.L. He, Enhancing extraordinary transmission of light through a metallic nanoslit with a nanocavity antenna, Opt. Lett. 34 (1) (2009) 16-18.

[7] X.F. Li, S.F. Yu, Long-wavelength optical transmission of extremely narrow slits via hybrid surfaceplasmon and Fabry-Perot modes, J. Appl. Phys. 108 (1) (2010) 013302

[8] X.Y. He, X.N. Fu, Y.W. Luo, Analysis of the extraordinary transmission properties of arrays of sub-wavelength holes on a metal film in the terahertz region, J. Mod. Opt. 56 (15) (2009) 1698-1703.

[9] C.M. Wang, Y.C. Chang, Reflection and emission properties of an infrared emitter, Opt. Express 15 (22) (2007) 14673-14678.

[10] M.R. Gadsdon, I.R. Hooper, J.R. Sambles, Surface plasmon polaritons on deep, narrow-ridged rectangular gratings, J. Opt. Soc. Am. B 26 (6) (2009) 1228 1237.

[11] Y.Q. Ma, J. Zhou, Z.Y. Wang, Surface plasmon waves on structured metal surface with periodic grooves modified by perpendicular cuts, IEEE Photonic. Technol. L 22 (7) (2010) 450-452.

[12] Y.F. Liu, J. Kim, Polarization-diverse broadband absorption enhancement in thin-film photovoltaic devices using long-pitch metallic gratings, J. Opt. Soc. Am. B 28 (8) (2011) 1934-1939.

[13] M. Grande, R. Marani, F. Portincasa, G. Morea, V. Petruzzelli, A. D’Orazio, V. Marrocco, D. de Ceglia, M.A. Vincenti, Asymmetric plasmonic grating for optical sensing of thin layers of organic materials, Sens. Actuators, B 160 (1) (2011) 1056-1062.

[14] N. Kato, L. Lee, R. Chandrawati, A.P.R. Johnston, F. Caruso, Optically characterized DNA multilayered assemblies and phenomenological modeling of layer-by-layer hybridization, J. Phys. Chem. C 113 (50) (2009) 21185-21195.

[15] G. Bao, Z.M. Chen, H.J. Wu, Adaptive finite-element method for diffraction gratings, J. Opt. Soc. Am. B 22 (6) (2005) 1106-1114.

[16] A.j. Dhawan, S.J. Norton, M.D. Gerhold, T.V. Dinh, Comparison of FDTD numerical computations and analytical multipole expansion method for plasmonics active nanosphere dimmers, Opt. Express 17 (12) (2009) 96889703. 